INPLASY

PROTOCOL

To cite: Chen et al. Efficacy

and safety of acupoint

injection therapy for frozen

shoulder: a protocol of

systematic review and meta-

analysis. Inplasy protocol

202110026. doi:

10.37766/inplasy2021.1.0026

Received: 10 January 2021

Published: 10 January 2021

Corresponding author:

Chen Yue

1060003610@qq.com

Author Affiliation:

Hospital of Chengdu

University of Traditional

Chinese Medicine

Support: 100 Talents Program.

Review Stage at time of this submission: The review has not yet started.

Conflicts of interest:

None.

\section{Efficacy and safety of acupoint injection therapy for frozen shoulder: a protocol of systematic review and meta-analysis}

Chen, Y1; Hu, Y2; Mu, P3; Feng, J4; Qu, P5; Xiong, F6.

Review question / Objective: The purpose of this study is to evaluate the efficacy and safety of acupoint injection for frozen shoulder.

Condition being studied: Frozen shoulder, also known as adhesive capsulitis, is a painful condition that most commonly affects people in the sixth decade of life. The capsule of the shoulder joint becomes inflamed, then scarred and contracted, causing pain, stiffness, and loss of function. People with frozen shoulder can struggle with basic daily activities and have sleep disturbance due to shoulder pain, condition that is usually managed in the primary care setting with a combination of analgesics, injections, and physiotherapy. Studies have shown that acupoint injection is effective in the prevention and treatment of frozen shoulder. Acupoint injection therapy is a technique that injects corresponding human acupoints in Chinese and Western medicine to achieve the purpose of curing diseases. Through this therapy, acupoint penetration, drug efficacy and acupuncture stimulation can be more effective, and the clinical symptoms of frozen shoulder can be significantly improved. Thus, we intend to conduct a systematic review and meta-analysis to testify the efficacy and safety of acupoint injection therapy for frozen shoulder.

INPLASY registration number: This protocol was registered with the International Platform of Registered Systematic Review and Meta-Analysis Protocols (INPLASY) on 10 January 2021 and was last updated on 10 January 2021 (registration number INPLASY202110026).

\section{INTRODUCTION}

Review question / Objective: The purpose of this study is to evaluate the efficacy and safety of acupoint injection for frozen shoulder.

Rationale: A lot of clinical studies have reported that acupoint injection has a good 
effect on the treatment of frozen shoulder, but there is no relevant systematic review or meta-analysis.

Condition being studied: Frozen shoulder, also known as adhesive capsulitis, is a painful condition that most commonly affects people in the sixth decade of life. The capsule of the shoulder joint becomes inflamed, then scarred and contracted, causing pain, stiffness, and loss of function. People with frozen shoulder can struggle with basic daily activities and have sleep disturbance due to shoulder pain, condition that is usually managed in the primary care setting with a combination of analgesics, injections, and physiotherapy. Studies have shown that acupoint injection is effective in the prevention and treatment of frozen shoulder. Acupoint injection therapy is a technique that injects corresponding human acupoints in Chinese and Western medicine to achieve the purpose of curing diseases. Through this therapy, acupoint penetration, drug efficacy and acupuncture stimulation can be more effective, and the clinical symptoms of frozen shoulder can be significantly improved. Thus, we intend to conduct a systematic review and meta-analysis to testify the efficacy and safety of acupoint injection therapy for frozen shoulder.

\section{METHODS}

Search strategy: CNKI, Wanfang, VIP , CBM, PubMed, Embase and Cochrane Library databases were searched for this study. Take the subject terms combined with free words to search, take PubMed as an example: terms consist of disease (frozen shoulder OR shoulder, frozen OR bursitis OR bursitis of shoulder ) and intervention (Acupoint Injection OR Acupuncture Point Injection OR point injection therapy OR Acupuncture Points) and research types (randomized controlled trial OR controlled clinical trial OR random trials).

Participant or population: Patients were diagnosed with frozen shoulder and the study belongs to randomized controlled trial. Clinical results included the university of California at Los Angeles shoulder rating scale (UCLA), constant-murley score, disabilities of the arm, shoulder and hand score (DASH), clinical effectiveness and Visual analog scale (VAS). The diagnostic criteria of frozen shoulder in the selected literature should meet the internationally recognized standards. Otherwise, studies will be excluded if they cannot meet the inclusion criteria.

Intervention: Interventions included treatment with acupoint injection. The drug types for acupoint injection, the acupuncture points for injection, treatment frequency, and duration of treatment will not be restricted. The use of acupoint injection is the only difference between intervention and control.

Comparator: The control interventions will include: positive interventions, placebo, no intervention. The choice of specific forms are as follows: (1). acupoint injection VS positive interventions; (2). acupoint injection + positive interventions VS positive interventions; (3). acupoint injection VS placebo; (4). acupoint injection VS no intervention.

Study designs to be included: All relevant randomized controlled trial OR controlled clinical trial OR random trials will be included.

Eligibility criteria: Patients meeting the diagnostic criteria of frozen shoulder. There will be no restrictions on the length of treatment and duration of follow-up. The language of the trials to be included only Chinese or English. Without any date of dissemination or restriction of publication type.

Information sources: CNKI, Wanfang, VIP, CBM, PubMed (including MEDLINE), Embase, Cochrane Library databases and Web of Science (Core Collection) were searched for this study.

Main outcome(s): The primary outcome is UCLA score and DASH score. 
Additional outcome(s): The secondary outcomes are clinical effectiveness, constant-murley score, VAS and the incidence of adverse reaction.

Data management: (1) EndnoteX9 and Excel software will be used to extract data, and the content will be stored in electronic chart. (2) Different researchers will separately screen the titles and abstracts of records acquiaed potential eligibility which comes from the electronic databases. Full texts screening and data extraction will be conducted afterwards independently. Any disagreement will be resolved by discussion until consensus is reached or by consulting a third author, in this step, we will use Endnote. (3) The following data will be extracted: author, year of publication, country, interventions of experimental groups and control groups, time point, outcome measures, age of patients, total number of people included in the study, patients' basic information, etc. Different researchers will separately extract data. Any disagreement regarding data extraction will be will be resolved by discussion until consensus is reached or by consulting a third author. In this step, we will use Excel.

Quality assessment / Risk of bias analysis: Two reviewers will independently assesses the quality of the selected studies according to the Cochrane Collaboration's tool for randomized controlled trials. Items will be evaluated in three categories: Low risk of bias, unclear bias and high risk of bias. The following characteristics will be evaluated: random sequence generation (selection Bias), allocation concealment (selection bias), blinding of participants and personnel (performance bias), incomplete outcome data (attrition bias), selective reporting(reporting bias)and other biases. Results from these questions will be graphed and assessed using Review Manager. The results will be presented in the form of a graph, and will be independently evaluated by two researchers. If there are differences of opinion, they will be discussed with the third researcher.
Strategy of data synthesis: We will use RevMan software to perform the metaanalysis. We will summarize data using risk ratios (RR) with $95 \% \mathrm{Cl}$ for binary outcomes or mean difference (MD) with $95 \% \mathrm{Cl}$ for continuous outcomes. $\mathrm{I}^{2}$ value and $P$-value will be used to test the degree of heterogeneity. When $\mathrm{P}>0.1, \mathrm{I}^{2}<50 \%$, no heterogeneity was considered between the studies and the fixed effect model will be used for statistical analysis; otherwise, the random effect model will be used. If there was significant clinical heterogeneity between studies, only descriptive analysis was performed. For a study with incomplete result data, we will try to contact the first author.

Subgroup analysis: There is no presubgroup plan for this project. When there are significant differences, we will carry out subgroup analysis according to the control group intervention measures and different results.

Sensibility analysis: Sensitivity analysis will be conducted by alteration of the analysis model, selection of effect size, and exclusion of individual articles.

Language: Chinese.

Country(ies) involved: China.

Keywords: frozen shoulder, acupoint injection, systematic review, meta-analysis protocol.

Dissemination plans: We plan to publish a systematic review based on this protocol.

Contributions of each author:

Author 1 - Chen Yue - Conceptualization.

Author 2 - Hu Yimei.

Author 3 - Mu Panyun.

Author 4 - Feng Jie.

Author 5 - Qu Peihua.

Author 6 - Xiong Feng. 